\title{
RADIO OBSERVATIONS OF THE COMET AREND-ROLAND
}

\author{
G. R. WHITFIELD AND J. HÖGBOM \\ Mullard Radio Astronomy Observatory, Cavendish Laboratory \\ Cambridge, England
}

Observations were made at Cambridge between 1957 March 12 and May 13 in an attempt to detect radio emission from the comet at 3.7 - and $7.9-\mathrm{m}$ wavelengths by observing radio stars through its tails, to derive information on the electron density in the manner used by Hewish [1] in investigating the outermost layers of the solar corona.

Five different instruments were used including two interferometers of large resolving power, which enabled a very low limit to be placed on the radio emission from the head. The possibility of radio emission from the extended tail was investigated with interferometers of smaller resolving power.

In no case was any detectable effect observed; the upper limits of the flux density are given in Table I. Solar emission prevented the observation of three comparatively intense radio stars through the comet's tail, but it was possible to show that there was no detectable scattering of emission from weaker stars greater than the resolution of the interferometers. It may therefore be concluded that the irregular variations of the electron density in the tail of the comet were insufficient to produce scattering as great as onehalf degree at $3.7-\mathrm{m}$ wavelength or one degree at $7.9-\mathrm{m}$ wavelength.

The magnitude of the scattering produced by an irregular region depends on $\Delta N$, the root-mean-square departure of electron density from the mean value and $l$, the scale of the irregular structure.

TABLE I

Wavelength
(meters)

3.7

7.9

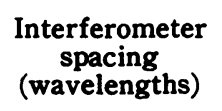

120

10

5

60

10

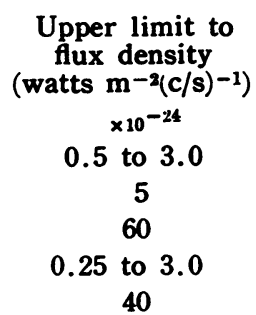

The present observations indicate that $\Delta N / \sqrt{ } T$ is less than 100 , when $\Delta N$ is measured in electrons $/ \mathrm{cm}^{3}$ and $l$ in kilometers. 
NO. 9

Optical photographs of the tail of the comet show structure as fine as $10,000 \mathrm{~km}$, leading to an upper limit of $10^{4}$ electrons $/ \mathrm{cm}^{3}$ for $\Delta N$.

Further information concerning the observations is given in a paper published by Whitfield and Högbom [2].

\section{REFERENCES}

[1] Hewish, A. Proc. Roy. Soc. A 228, 238, 1955.

[2] Whitfield, G. R., and Högbom, J. Nature, 180, 602, 1957. 